tion needed. The superposition can be conveniently achieved photographically by successive exposures on the same plate.

Research Laboratories,

V. VAND.

Lever Brothers and Unilever, Limited,

Port Sunlight, Cheshire.

${ }^{1}$ Nature, 152, 411 (1943).

2 Nature, 153, 81 (1944).

Nature, 154, 69 (1944).

\section{Elastic Constants of Diamond}

By using a suitably cut and silvered quartz or tourmaline wedge, we have been able to generate continuously varying ultrasonic frequencies. This forms the basis of a new method for measuring the effective elastic constant of a given plate. In this method, the particular frequency for which there is best transmission through the plate is located by allowing the waves to pass through the plate into a liquid and observing positions of maximum intensity in respect of the usual Debye-Sears pattern. This frequency $\nu$ is related to the effective elastic constant $C_{33}^{\prime}$ by the relation $4 v^{2} d^{2} \rho=C_{33}^{\prime}$, where $d$ and $\rho$ are respectively the thickness and the density of the plate.

By making such observations on an octahedral and a dodecahedral plate of diamond, two independent effective elastic constants have been obtained. It is not possible to get the third one by this method, but by combining the results with the known bulk modulus $5.9 \times 10^{12}$ dynes per sq. cm. (which represents the mean of observations by Adams ${ }^{1}$ and by Williamson $^{2}$ ), the following values expressed in dynes per sq. em. have been obtained for the elastic constants of diamond:

$C_{11}=9 \cdot 4 \times 10^{12} ; C_{12}=4.2 \times 10^{12} ; C_{44}=4.2 \times 10^{12}$.

These results are now reported in view of their importance, but their significance will be discussed and the details of the method given in a fuller paper in the Proceedings of the Indian Academy of Sciences. It may be remarked here that we have found these elastic constants to be in satisfactory agreement with the force constants derived from the known frequencies of the diamond lattice.

S. Bhagavantam.

J. Bhimasenachar.

Department of Physics,

Andhra University,

Guntur. Sept. 12.

${ }^{1}$ J. Wash. Acad. Sci., 11, 45 (1921).

3. Frank. Inst., 193, 491 (1922).

\section{A Reversible Contraction Phenomenon in Animal Hairs}

Asthough the action of cuprammonium hydroxide on cellulose has been studied in considerable detail, comparatively little is known of its effect on keratin, except that under certain conditions hair and wool can be dissolved in the reagent $t^{1,2}$. It is therefore of interest to describe some observations on the action of cuprammonium hydroxide on wool which promise to yield valuable information on the mechanism of long-range elasticity in animal hairs and on the structure of proteins.

If a wool fibre (Lincoln) is immersed in a solution prepared by dissolving purified copper hydroxide in concentrated ammonia solution, it will, after a time depending on the copper content of the solution, become stained greenish-blue and contract in length. Prolonged washing in water fails to remove the stain and has little effect on the length, although a further small contraction may occur. Under the correct conditions it is possible to realize in this way a contraction of 28 per cent of the initial length. If the fibre is now placed in a dilute solution of sulphuric acid for a few minutes, the blue stain disappears and the fibre returns practically to its original length. Subsequent washing in water produces no further change in colour or length. The power of the fibre to recover its original length depends on the severity of the treatment; after prolonged immersion in concentrated solutions the recovery may stop when the fibre is still some 10 per cent shorter than the initial length. Even though the fibre retains its power of recovery, it is weakened by the treatment with cuprammonium hydroxide, and evidently side-chain breakdown takes place in the solution.

The dimensional changes are accompanied by changes in the X-ray photograph, which becomes weaker and vaguer without loss of orientation or pronounced spacing changes, until, when the contraction is a maximum, it has almost disappeared. This is in striking contrast to the effects in ordinary supercontraction, where the normal $\alpha$-photograph may still be present for contractions of the order of 20 per cent, although the disoriented $\beta$-photograph may appear under certain conditions ${ }^{3}$. After acid treatment to remove the copper, the $\alpha$-photograph returns, comparable in every way with the original photograph. This sequence can be repeated several times.

The coloration of the fibres after treatment with cuprammonium hydroxide and washing in water is clearly due to the adsorption of copper. Our measurements showed that the amount of copper adsorbed under conditions corresponding to those giving maximum contraction is about 29 per cent (by weight, calculated on the dry weight of the fibre), the observed values lying between 26 and 32 per cent for different concentrations of the reagent. This corresponds approximately to one copper atom per two amino-acid residues. The total increase in weight (40 per cent) suggests that the copper is present as a complex.

The observed contraction may be attributed to the powerful attraction of the copper for the appropriate active groups (for example, amino- and iminogroups) in the keratin complex. This contraction would be resisted by those side-chain cross-linkings between the polypeptide chains which are not broken by the reagent itself, and at first enough would be left stressed but unbroken to assure the return of the fibre to the initial length on removal of the copper. Prolongation of the treatment, however, would ultimately reduce the number of unbroken erosslinkings below the minimum required for complete recovery.

We have observed a similar phenomenon in fibres treated with solutions of some, but not all, related copper compounds.

\section{S. WHEWELL.} H. J. Woons.

Department of Textile Industries, University, Leeds. Sept. 11.

1 Bergmann, M., D.R.P., 445,503.

2Rimington, C., and Wool Industries Research Association, Brit.Pat. 343,838 .

${ }^{3}$ Astbury, W. T., and Woods, H. J., Phil. Tran8., A, 232, 333 (1933). 